\title{
Balanced RM2: An Improved Data Placement Scheme for Tolerating Double Disk Failures in Disk Arrays ${ }^{\star}$
}

\author{
Dae-Woong Kim, Soon-Ho Lee, and Chan-Ik Park \\ Department of Computer Science and Engineering/PIRL \\ Pohang University of Science and Technology \\ Pohang, Kyungbuk 790-784, Republic of Korea \\ cipark@postech.ac.kr
}

\begin{abstract}
There is a growing demand for high data reliability beyond what current RAIDs can provide and users need various levels of data reliability. In order to meet these requirements, an efficient data placement scheme called RM2 has been proposed in 11, which enables a disk array system to tolerate double disk failures. However, RM2 has high data reconstruction overhead due to data dependency between parity groups in the case of double disk failures, suffering from large performance fluctuation according to disk failure patterns. To minimize the reconstruction overhead, this paper presents an improved data placement scheme called Balanced RM2. Experimental results show that the performance fluctuation of the Balanced RM2 becomes much less than that of the RM2 regardless of read or write. It is also shown that Balanced RM2 provides better performance than RM2 by $5 \sim 20 \%$ in the case of double disk failures.
\end{abstract}

\section{Introduction}

There has been much research on improving I/O performance, known as data declustering and disk striping, in disk array systems 2/344. However, incorporating a large number of disks into a disk array system makes the system more prone to failure than a single disk would 3 .

In general, high reliability can be achieved by redundant information. For example, RAID (Redundant Arrays of Independent Disks) enables a disk array system to tolerate a single disk failure by maintaining parity information. In order to meet reliability requirement beyond what RAID can provide, that is, tolerate more than one disk failures, there has been much research on high reliability of disk arrays [156]. Blaum et al. have proposed EVENODD in [6]. This

\footnotetext{
* The authors would like to thank the Ministry of Education of Korea for its support toward the Electrical and Computer Engineering Division at POSTECH through its BK21 program. This research was also supported in part by HY-SDR IT Research Center and in part by grant No. R01-2003-000-10739-0 from the Basic Research Program of the Korea Science and Engineering Foundation.
} 
method maintains two types of redundant information: horizontal redundancy and diagonal redundancy. Note that EVENODD requires only two additional disks (which is optimal). However, EVENODD has an apparent restriction; the number of information disks has to be a prime number. Park has proposed RM2 in 1] which does not exhibit any explicit I/O bottleneck by enforcing the even distribution of parity information across all disks. However, RM2 has high data reconstruction overhead due to data dependency between parity groups in the case of double disk failures, suffering from large performance fluctuation according to disk failure patterns.

This paper presents an improved data placement scheme called Balanced $R M 2$ which distributes the load of data reconstruction as evenly as possible over all the remaining disks under disk failures.

The rest of the paper is organized as follows. Section 2 describes the RM2 architecture. Section 3 presents the Balanced RM2 architecture and its data reconstruction method. Section 4 gives experimental results for various cases. Finally, concluding remarks and future works are given in Section 5

\section{Backgrounds}

A disk array is assumed to consist of $N$ disks each of which handles independent requests in parallel. The address space of a disk array is logically structured with a set of stripe units. A stripe unit is a basic unit of data striping consisting of a group of logically contiguous blocks. It is placed consecutively on a single disk before placing another stripe unit on a different disk. A stripe consists of $N$ stripe units each of which has the same physical address in each disk. If a stripe contains only data units, then it is called a data stripe. If a stripe consists of parity units only, then it is called a parity stripe. A parity group is defined as a set of data units and a parity unit, where the parity unit is computed from the set of data units. We define a stripe group as a set of data stripes and a parity stripe, which covers all stripe units in a given parity group. In RM2, data and parity mapping of a stripe group are defined by an $N \times N$ redundancy matrix. In the redundancy matrix, each column corresponds to a disk and each row corresponds to a parity group. Each column has one entry with a value of -1 and two entries with a value of $k$ for $1 \leq k \leq M-1$ where $M$ is the size of a stripe group. Let $R M_{i, j}$ be an element of the redundancy matrix with a value ranging from -1 to $M-1$. According to the value of $R M_{i, j}$, the interpretation differs as follows:

$-R M_{i, j}=-1$ : A parity unit of the disk $j$ belongs to the parity group $i$.

$-R M_{i, j}=0$ : No meaning.

$-R M_{i, j}=k$ for $1 \leq k \leq M-1$ : The $k$-th data unit of the disk $j$ belongs to the parity group $i$.

Figure 11 represents the data layout of the RM2 architecture and its corresponding $7 \times 7$ redundancy matrix when the number of disks is seven. Note that each disk in RM2 has equal amount of parity information. There are two data 


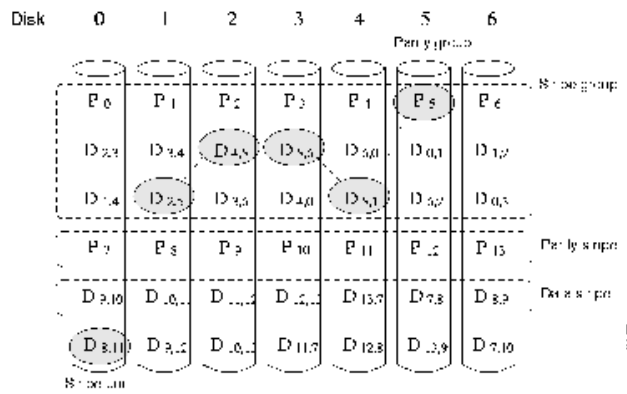

(4) Data layoul

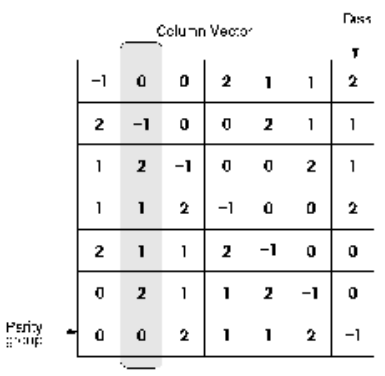

(b) Reduriduricy matrix

Fig. 1. Data layouts of RM2 and its redundancy matrix for $N=7$ and $M=3$ where $N$ is the number of disks and $M$ is the size of a stripe group

stripes and one parity stripe in a stripe group, i.e., $M=3$. The amount of disk space used to store parity information is represented by the reciprocal of the size of a stripe group (i.e., $1 / M)$, called redundancy rate. For example in Figure 1 the redundancy rate is $1 / 3$ since a stripe group consists of two data stripes and one parity stripe, i.e., the size of a stripe group is 3. $P_{i}$ represents the parity unit in the $i$-th parity group and $D_{i, j}$ means that the data unit is involved in computing parity information of both the $i$-th and $j$-th parity groups. For example, in order to compute the parity unit $P_{5}$, we have to consider the four data units $D_{2,5}, D_{4,5}, D_{5,6}$, and $D_{5,1}$. And, the data unit $D_{8,11}$ belongs to both parity groups $P_{8}$ and $P_{11}$.

\section{Balanced RM2}

The main problem of RM2 is that it has high data reconstruction overhead due to data dependency between parity groups in the presence of double disk failures. To minimize the reconstruction overhead, this section describes how to place data and parity to address the data dependency problem.

\subsection{Dual-Symmetry}

In RM2, c-independence was introduced to formulate how to place In order to deal with the efficiency of data reconstructing operation, we define dualsymmetry of RM2.

Definition 1 (Dual-Symmetry). The column vector of a redundant matrix is dual-symmetric if every positive entries are symmetrically positioned both around the entry with a value of -1 (parity unit) and around a sequence of zero entries simultaneously. And the redundant matrix is said to be dual-symmetric if its column vector is dual-symmetric. 


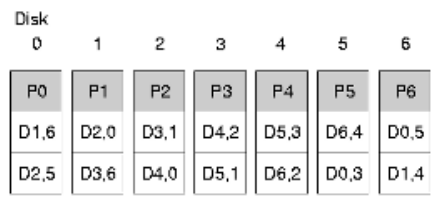

(a) Data layout.

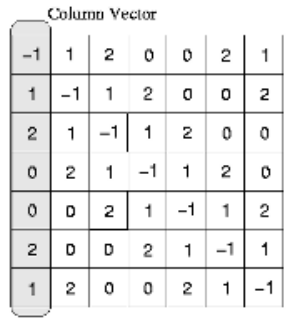

(b) Redundancy matrix

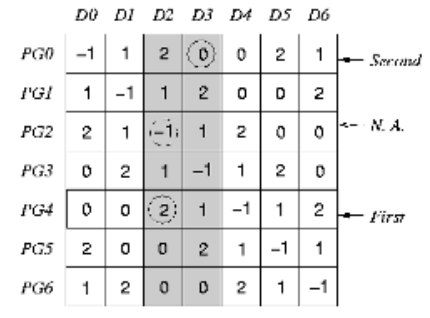

(c) Recovery Sequence of $\mathrm{D} 4,2=\{\mathrm{PG} 4, \mathrm{PG} 0\}$

Fig. 2. Data layouts of the Balanced RM2 and its redundancy matrix for $N=7$ and $M=3$

For example, Figure 1(b) shows a redundancy matrix that is c-independent, but whose column vector is not dual-symmetric. Figure 2(b) represents the redundancy matrix that is both c-independent and dual-symmetric.

For the Balanced RM2 architecture, it is necessary for a redundancy matrix to meet the following two conditions:

- any pair of columns is c-independent.

- the column vector is dual-symmetric.

The first condition guarantees that all failed data blocks can be reconstructed in the case of one or two disk failures [1. And, the second condition enforces that the load of data reconstruction are evenly distributed, which will be described in detail later.

Figure 2 represents the data layout of the Balanced RM2 architecture and its redundancy matrix when $N=7$ and $M=3$. It looks similar to that of RM2 (see Figure1), but note that all stripe units of a parity group are symmetrically positioned around the parity unit.

\subsection{Recovery Points and Sequences}

A recovery point is defined as a parity group that has only one inaccessible data unit owing to disk failures. If the inaccessible data unit is a parity unit, then the recovery point will not be used as a starting sequence for data reconstruction. Otherwise, it can be regarded as a starting sequence for data reconstruction. A recovery path is defined as the sequence of all parity groups reachable from a recovery point. For example shown in Figure 2(c), parity groups $P G 0$ and $P G 5$ become recovery points when disks $D 2$ and $D 3$ fail. Thus, two recovery paths $\{P G 0, P G 4, P G 2\}$ and $\{P G 5, P G 1, P G 3\}$ are found. If a recovery path ends up with another recovery point, then it is called a bi-directional recovery path. Otherwise, it is called a uni-directional recovery path.

A recovery sequence is defined as the shortest recovery path which starts from a recovery point and ends up with a parity group including the failed data unit. Following the recovery sequence, we can reconstruct the failed data unit. The length of a recovery sequence represents the number of parity groups involved in 
Table 1. The length of minimum and maximum recovery sequence depending on the distance between two failed disks, where $N=7$ and $M=3$

\begin{tabular}{|c||c|c||c|c|}
\hline \hline \multirow{2}{*}{$\begin{array}{c}\text { Distance between } \\
\text { two failed disks }\end{array}$} & \multicolumn{2}{c||}{ RM2 } & \multicolumn{2}{c|}{ Balanced RM2 } \\
\cline { 2 - 5 } & minimum & maximum & minimum & maximum \\
\hline \hline 1 & 1 & 4 & 1 & 2 \\
\hline 2 & 1 & 2 & 1 & 1 \\
\hline 3 & 1 & 1 & 1 & 1 \\
\hline 4 & 1 & 1 & 1 & 1 \\
\hline
\end{tabular}

the recovery sequence. For example, the recovery sequence length in RAID5 is one, that is, accessing only one parity group is enough for data reconstruction.

For example shown in Figure 2 consider the case where we want to reconstruct the first data unit, $D_{4,2}$, in the disk $D 3$ when the disks $D 2$ and $D 3$ fail (denoted as shaded columns). Both the parity group PG2 and PG4 are the parity groups that include the failed data unit $D_{4,2}$ since $R M_{2,3}$ and $R M_{4,3}$ have a value of one in the redundancy matrix. From the parity group $P G 4$, we can find the second data unit of $D 2, D_{4,0}$, as a pivot because $P G 4$ has another failed data unit. Then, the recovery sequence includes the parity group $P G 0$ because $P G 0$ also depends on the data unit selected as a pivot. Since $P G 0$ has only one broken data unit, $P G 0$ is then a recovery point that can be a starting sequence of data reconstruction. Thus, we get the recovery sequence that includes the parity group $P G 4$ and $P G 0$. However, from the parity group $P G 2$, we cannot further construct a recovery sequence because the pivot for the parity group $P G 2$ is a parity unit.

We know that the length of a recovery sequence has effects on the data reconstruction performance in the case of disk failures.

Lemma 1. The Balanced RM2 has at least two recovery points in the case of double disk failures.

Theorem 1. The length of any recovery sequence in the Balanced RM2 is at most $M-1$, where $M$ is the size of a stripe group.

For the proof of Lemma 1 and Theorem 1, please refer to [7].

Consequently, the Balanced RM2 architecture is expected to provide better performance than RM2 in the case of double disk failures since the worst case of recovery sequence lengths in the RM2 is $2(M-1)$ while only $M-1$ in Balanced RM2, where $M$ is the size of a stripe group. Table 1 shows the minimum and maximum recovery sequence depending on the distance between two failed disks.

\section{Performance Evaluation}

This section evaluates the performance, in terms of throughput and response time, of RM2, EVENODD, and Balanced RM2 under a highly concurrent workload of small reads and writes. 
Table 2. Disk operation overheads in each disk array architecture according to the number of disk failures. $C_{R}$ and $C_{W}$ represent the cost of read operations and write operations, respectively. $N$ denotes the number of disks and $G$ represents the size of a parity group. $L$ and $L^{*}$ denote the number of parity groups involved in reconstructing the failed data unit in RM2 and Balanced RM2 each other

\begin{tabular}{|c||c|c||c|c|}
\hline \hline \multirow{2}{*}{ Disk arrays } & \multicolumn{2}{c||}{ Single failure } & \multicolumn{2}{c|}{ Double failures } \\
\cline { 2 - 5 } & Read cases & Write case & Read case & Write case \\
\hline EVENODD & $(N-1) C_{R}$ & $(N-1) C_{R}+2 C_{W}$ & $2(N-1) C_{R}$ & $2(N-1) C_{R}+2 C_{W}$ \\
\hline RM2 & $(G-1) C_{R}$ & $(G-1) C_{R}+2 C_{W}$ & $L(G-1) C_{R}$ & $L(G-1) C_{R}+2 C_{W}$ \\
\hline Balanced RM2 & $(G-1) C_{R}$ & $(G-1) C_{R}+2 C_{W}$ & $L^{*}(G-1) C_{R}$ & $L^{*}(G-1) C_{R}+2 C_{W}$ \\
\hline
\end{tabular}

Simulation Environments. Our experiments were run on RAIDframe [8] a simulator in which various RAID architectures can be modeled and evaluated. RAIDframe's synthetic workload generator makes highly concurrent sequences of user I/O requests for disk accesses from multiple client threads. Each individual user request is converted into a set of read and write accesses on the specific disk in the disk array.

The individual disk of the array was modeled with HP 2247 whose revolution time, single cylinder seek time and average seek time are $11.12 \mathrm{~ms}, 2.5 \mathrm{~ms}$ and $10 \mathrm{~ms}$, respectively. The stripe unit was configured to be of 8 sectors, i.e., 4 Kbytes. For disk queue management, the shortest-seek-time-first (SSTF) queueing policy is adopted in a disk queue that can hold 10 outstanding requests. The synthetic workload generator incessantly makes random and independent 4 Kbyte accesses aligned to the stripe unit boundaries. These workloads are chosen because small requests are typically the most demanding for many RAID architectures 91011].

Each disk array is configured as $N=15$ and $M=3$. The experiments are performed according to the increase of client threads, which shows us the saturation point of throughput in each disk array architecture.

Analytic Comparison. Table 2 shows how many disk operations are needed to serve small-sized user requests in each architecture depending on the number of disk failures. Note that each array has different values in the size of a parity group as well as the length of a recovery sequence. For example, the size of a parity group in the RM2 architecture is the same as that in the Balanced RM2 architecture whereas the RM2 has the longer recovery sequence than the Balanced RM2.

Comparison under Double Disk Failure. In the case of read operations, it is expected that the RM2 and the Balanced RM2 have much better performance than the EVENODD due to the declustering effect [10. In detail, the Balanced RM2 architecture provides better performance than the RM2 architecture since it needs smaller number of parity groups in reconstructing the failed data unit. Figure 3(a) shows that the Balanced RM2 architecture reaches 


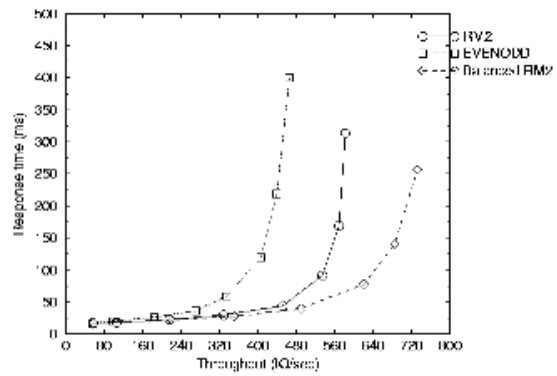

(a) Reads

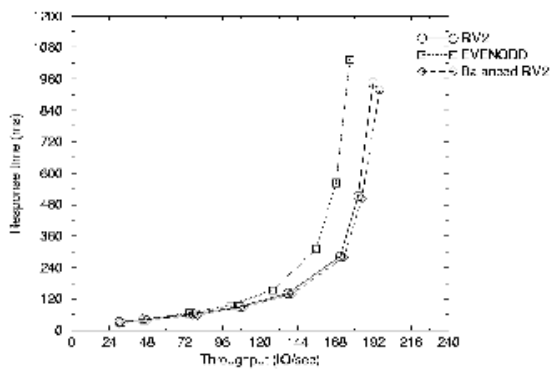

(b) Writes

Fig. 3. Performance comparison for the case of double disk failures

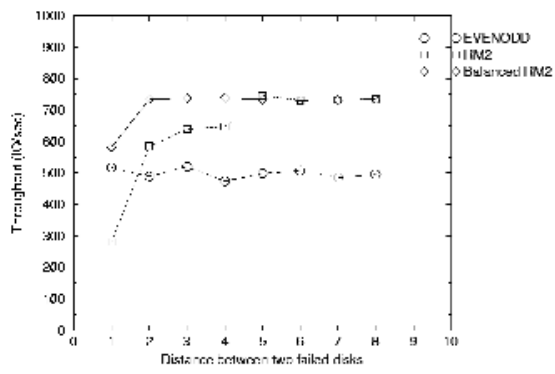

(a) Rcads

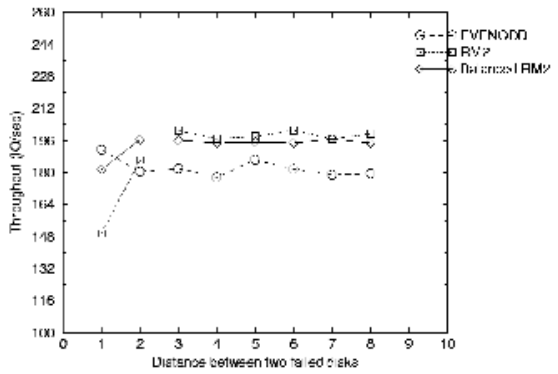

(b) Writes

Fig. 4. Throughput according to the distance between two failed disks

its point of saturation at about 740 reqs/sec whereas the RM2 architecture at about $580 \mathrm{reqs} / \mathrm{sec}$ and the EVENODD architecture at about $460 \mathrm{reqs} / \mathrm{sec}$.

In the case of the write operations, RM2 and Balanced RM2 have non trivial performance advantage over EVENODD as shown in Figure 3(b). Furthermore, the performance of Balanced RM2 is slightly better than RM2 due to the smaller number of parity groups involved in reconstructing the failed data unit. In the case of write operations, the Balanced RM2 architecture reaches its point of saturation at about $194 \mathrm{reqs} / \mathrm{sec}$, compared to about $190 \mathrm{reqs} / \mathrm{sec}$ for the RM2 architecture and 175 reqs/sec for the EVENODD architecture.

\section{Comparison According to the Distance between Two Failed Disks.}

Figure 4 demonstrates how the distance between two failed disks affects on the performance of read and write operation in the case of double disk failures. It is shown that Balanced RM2 provides a better throughput than RM2. Remember that the maximum length of a recovery sequence in the Balanced RM2 is $(M-1)$ whereas $2(M-1)$ in RM2. The Balanced RM2 architecture has better performance than the EVENODD architecture because of the declustering effect. However, the declustering effect becomes negligible due to long recovery sequences in the case of adjacent disk failures. 


\section{Conclusions}

This paper has presented an improved data and parity placement scheme called Balanced RM2 to minimize high data reconstruction overhead in RM2. Minimizing data reconstruction overhead is achieved by reducing the number of parity groups involved in reconstructing the failed data unit.

The experiments have been conducted by the RAIDframe under a highly concurrent workload of small-sized reads and writes. It has been shown that the Balanced RM2 provides better performance than the RM2 and the EVENODD in data reconstruction operations.

The data and parity placement schemes using the redundancy matrix are applicable to many research fields for disk arrays. Currently, we are considering the data and parity placement schemes to tolerate $n$ disk failures in disk arrays.

\section{References}

1. Park, C.I.: Efficient placement of parity and data to tolerate two disk failures in disk array systems. IEEE Transactions on Parallel and Distributed Systems 6 (1995) 1177-1184

2. Kim, M.Y., Tantawi, A.N.: Asynchronous disk interleaving. IEEE Transactions on Computers 40 (1991)

3. Patterson, D.A., Gibson, G.A., Katz, R.H.: A case for redundant array of inexpensive disks (raid). In: ACM SIGMOD Conference Proceedings, Chicago, Illinois (1988)

4. Narasimha, R.A.L., Banerjee, P.: An evaluation of multiple-disk i/o systems. IEEE Transactions on Computers 38 (1989) 1680-1690

5. Alvarez, G.A., Burkhard, W.A., Cristian, F.: Tolerating multiple failures in raid architectures with optimal storage and uniform declustering. In: Proceedings of the 24th Annual ACM/IEEE International Symposium on Computer Architecture(ISCA ‘97), Denver, Colorado (1997)

6. Blaum, M., Brady, J., Bruck, J., Menon, J.: Evenodd: An efficient scheme for tolerating double disk failures in raid architectures. IEEE Transactions on Computers 44 (1995)

7. Kim, D.W., Lee, S.H., Park, C.I.: Balanced rm2: An improved data placement scheme for tolerating double disk failures in disk arrays. Technical Report CSESSL-2003-08, System Software Lab., Department of Computer Science and Engineering, Pohang University of Science and Technology (2003)

8. Courtright, W.A., Gibson, G., Zelenka, J.: Raidframe: Rapid prototyping for disk arrays. In: Proceedings of the 1996 Conference on Measurement and Modeling of Computer Systems (SIGMETRICS). Volume 24. (1996) 268-269

9. Stodolsky, D., Gibson, G., Holland, M.: Parity logging: Overcoming the small write problem in redundant disk arrays. In: Proceedings of International Symposium on Computer Architecture, San Diego CA (1993)

10. Holland, M., Gibson, G.A.: Parity declustering for continuous operation in redundant disk arrays. Journal of Distributed and Parallel Databases 2 (1994)

11. Courtright, W.A., Gibson, G., Zelenka, J.: A structured approach to redundant disk array implementation. In: Proceedings of the International Symposium on Performance and Dependability. (1996) 